\title{
Proceso enseńanza-aprendizaje en investigación formativa. Experiencia de la Facultad de Educación Física Militar ${ }^{1}$
}

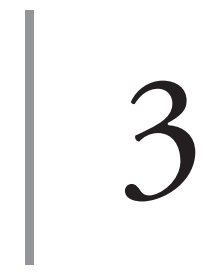

https://doi.org/10.21830/9789585380202.03

\author{
Ana Isabel García Muñoz \\ Paula Janyn Melo Buitrago ${ }^{3}$ \\ Escuela Militar de Cadetes "General José María Córdova”
}

Resumen. El objetivo del capítulo es describir la experiencia del proceso de enseñanza-aprendizaje en investigación formativa de la Facultad de Educación Física Militar de la Escuela Militar de Cadetes “General José María Córdova” (Esmic). Con este propósito se emplea una metodología cualitativa, tipo estudio de caso en el que se exploraron documentos y registros de la Facultad de los últimos cinco años para identificar casos en los cuales el aprendizaje en investigación formativa fue significativo. Los resultados del estudio evidencian que el proceso enseñanza-aprendizaje ha promovido el aprendizaje significativo en investigación formativa. Cada vez se observa mayor empoderamiento de los cadetes y alféreces en su proceso de formación. Las competencias investigativas adquiridas han sido principalmente en pensamiento crítico, escritura y comunicación. Se concluye que el nivel de formación y la experiencia docente en investigación son claves a la hora de orientar a los estudiantes hacia la resolución de preguntas e investigación.

Palabras clave: educación; educación física militar; investigación formativa; teorías del aprendizaje.

1 Este capítulo de libro parte de la investigación realizada por el Centro de Investigación de la Cultura Física (CICFI), de la Escuela Militar de Cadetes “General José María Córdova”. Los puntos de vista que se presentan en el capítulo pertenecen a las autoras y no reflejan necesariamente los de las instituciones participantes.

2 Magíster en Desarrollo Educativo y Social. Especialista en Rehabilitación Cardiopulmonar y en Docencia Universitaria. Terapeuta respiratoria. Investigadora júnior reconocida por Minciencias. Asesora del área Cardiopulmonar del Centro de Investigación de la Cultura Física (CICFI), de la Escuela Militar de Cadetes “General José María Córdova”. OrCid: https://orcid.org/0000-0003-4455-4534 - Contacto: ana. garcia@esmic.edu.co

3 Magíster en Educación con Énfasis en Pedagogía del Entrenamiento Deportivo. Especialista en Docencia Universitaria y licenciada en Educación Física. Coordinadora de Investigaciones de la Facultad de Educación Física Militar y docente universitaria en el área de entrenamiento deportivo e investigación aplicada a la educación física. OrCID: https://orcid.org/0000-0002-3492-7985 - Contacto: paula.melo@esmic.edu.co 


\section{Introducción}

El proceso enseñanza-aprendizaje hace referencia al conjunto de métodos y dinámicas que se generan entre los profesores y los estudiantes, en un contexto determinado, en pro de la adquisición del conocimiento. Al estudiar este proceso, de acuerdo con Borja et al. (2017), es necesario comprender los tres subsistemas que lo conforman: (1) el axiológico, que aporta valores y principios; (2) el psicológico, que se centra en el estudiante, su personalidad y comportamiento, y, finalmente, (3) el socio-histórico, que partiendo de la comprensión de la realidad del ahora, enfoca el proceso hacia su reconfiguración dentro de una sociedad que evoluciona constantemente (Borja et al., 2017).

\section{Marco teórico}

Para que el proceso educativo permita la aprehensión, procesamiento, comprensión y aplicación de lo aprendido a la vida práctica, es necesario que se genere un ambiente motivacional, en el que se susciten dinámicas que permitan una comunicación dialógica entre las partes, por medio de estrategias que promuevan situaciones de aprendizaje explícito e implícito, que a su vez conduzcan a la generación de competencias en los estudiantes, con miras a lograr un desempeño académico que se proyecte en un futuro cambiante y desafiante (Morales-Ocaña \& Higueras-Rodríguez, 2017), con lo cual se da cuenta de un aprendizaje significativo.

El constructivismo es el pilar fuerte en el que se fundamenta el aprendizaje significativo, toda vez que este último permite el enlace de lo aprendido con nuevos aprendizajes, a través del autoaprendizaje y del aprendizaje en interacción con otros. En este orden de ideas y desde la perspectiva de Suárez, citado por Parra (2014), corresponde al docente ser promotor de cambios y gestor de escenarios que faciliten la participación de los estudiantes en experiencias de discusión académica, desde una perspectiva crítica, en donde se les reconozca como sujetos activos, que aportan en la construcción de nuevo conocimiento. Para esto se requiere el análisis de sus ritmos de aprendizaje y la comprensión de las condiciones particulares que facilitan o entorpecen su proceso, al tiempo que el estudiante, al empoderarse de su compromiso en el proceso, se hace responsable y se autogestiona (Lizarazo \& Paredes, 2015; Parra, 2014). 
En el marco de esta realidad, la Escuela Militar de Cadetes "General José María Córdova” (EsMIC), desde siempre comprometida con la formación integral de los futuros oficiales del Ejército Nacional, ha venido modelando su Proyecto Educativo Institucional (PEI) (EsMic, 2016). Es así que incorpora el constructivismo como método de enseńanza-aprendizaje, con lo cual rompe esquemas tradicionalistas al tiempo que ubica la investigación formativa como eje transversal del proceso (Cabrera \& Bernal, 2018). Desde este lineamiento, el Proyecto Educativo del Programa (Pep) de Educación Física Militar se articula y promueve la adquisición de competencias, que ligadas a los valores institucionales, generan gusto por la investigación (Consejo de Facultad de Educación Física Militar, 2019).

A la fecha existen varias publicaciones que develan resultados del aprendizaje significativo, como resultado de experiencias en el aula, en asignaturas propias del área de conocimiento y del objeto de estudio de cada profesión. Sin embargo, no sucede lo mismo cuando se habla de asignaturas transversales como la investigación formativa. El objetivo de este estudio es describir la experiencia del proceso de enseñanza-aprendizaje en investigación formativa de la Facultad de Educación Física Militar para visibilizar si este proceso generó un aprendizaje significativo en los cadetes y alféreces.

\section{Método}

Se trata de un estudio cualitativo, tipo estudio de caso, con un enfoque constructivista y método hermenéutico, que buscó comprender y explicar el proceso de enseñanza-aprendizaje en investigación formativa de la Facultad de Educación Física Militar de los últimos cinco años.

Como la vinculación del modelo constructivista en la EsMic tuvo lugar en los últimos cinco años, también se realizó un análisis interpretativo y comparativo de las mismas categorías antes y después de dicha vinculación. En este análisis, los investigadores, como individuos partícipes del proceso, adoptaron una posición inductiva para describir y comprender la evolución del proceso y su influencia en las experiencias de éxito de los estudiantes. Para recolectar los datos, que fueron obtenidos de los documentos estudiados, se partió de la formulación de cinco categorías de estudio: (1) enfoques pedagógicos y (2) estrategias motivacionales, (3) competencias estudiantiles, (4) 
aprendizaje significativo (experiencias de éxito valoradas en la vinculación a semilleros) y (5) docentes.

En el análisis y la validación de la información se recurrió a la triangulación, con lo cual se buscó observar las similitudes o diferencias (Hernández et al., 2010), en este caso, de los documentos. Para esto se usó el modelo propuesto por Erzberger y Prein (1997), citados por Bonilla-Castro y Rodríguez (2005), en el cual "los hallazgos empíricos se integran bajo unos supuestos teóricos comunes que han debido ser desarrollados desde antes del comienzo de la investigación” (p. 285). De este modo, se trianguló la información obtenida de la revisión documental con el referente teórico en aprendizaje significativo, constructivismo e investigación formativa y la postura de los investigadores.

\section{Resultados}

A continuación, la tabla 1 presenta los archivos y documentos institucionales consultados por las investigadoras.

Tabla 1. Archivos y documentos institucionales consultados

\begin{tabular}{|c|c|}
\hline No & Título \\
\hline 1 & Documento maestro del programa para la obtención del registro calificado. \\
\hline 2 & $\begin{array}{l}\text { Acuerdo N.o } 008 \text { del } 19 \text { de febrero de 2016, por medio del cual se aprueba el } \\
\text { Pei de la Esmic. }\end{array}$ \\
\hline 3 & Pep del Programa de Educación Física Militar 2019. \\
\hline 4 & $\begin{array}{l}\text { Acuerdo N.o } 004 \text { del } 6 \text { de febrero de 2016, por medio del cual se reglamentan } \\
\text { los semilleros. }\end{array}$ \\
\hline 5 & $\begin{array}{l}\text { Resolución N.o } 0058 \text { del } 18 \text { de febrero de } 2016 \text {, por medio de la cual se regla- } \\
\text { mentan las opciones de grado. }\end{array}$ \\
\hline 6 & Repositorio de trabajos de grado de la Facultad de Educación Física Militar. \\
\hline 7 & Informe final de la renovación de la acreditación de la Facultad. \\
\hline 8 & $\begin{array}{l}\text { Documentos soporte del Comité de Ética y del Comité de Investigaciones de } \\
\text { la Facultad de Educación Física Militar. }\end{array}$ \\
\hline 9 & $\begin{array}{l}\text { Malla curricular extensa del Programa de Educación Física Militar integrada } \\
\text { con Ciencias Militares. }\end{array}$ \\
\hline 10 & $\begin{array}{l}\text { Syllabus de los saberes que trabajan en el aprendizaje de las competencias inves- } \\
\text { tigativas. }\end{array}$ \\
\hline
\end{tabular}

Fuente: Elaboración propia. 


\section{Primera categoría: Enfoques pedagógicos}

Se identificaron y lograron diferenciar las estrategias de enseñanza de la educación tradicional aplicada antes del año 2016, con las estrategias de enseñanza de una educación constructivista. Es así como antes del 2016, las estrategias del modelo educativo tradicionalista se evidenciaron en varios syllabus, en donde el docente y el contenido eran los ejes centrales de la acción educativa y donde se aplicaba con determinación el método expositivo o magistral.

Este modelo, además, estaba permeado por el sistema educativo castrense que preponderaba en el ámbito académico militar, el cual se caracterizaba por un proceso selectivo que enfatizaba los instrumentos evaluativos, más que los medios de enseñanza. En consecuencia, los estudiantes asignaban muy poca importancia a los contenidos que se les enseńaba, toda vez que de ellos solamente se esperaba participar de un prolongado proceso selectivo.

En tal sentido, hacia los ańos 2012 y 2013 se abrió paso a una mejora en los procesos realizados en el interior del Ejército, con miras al desarrollo de capacidades que respondieran a los nuevos retos y amenazas que se vislumbraban en un mundo cada vez más globalizado. Para ello, la institución planteó una serie de iniciativas que marcaron los lineamientos del desarrollo institucional, a través del fortalecimiento de los subsistemas de educación, doctrina, lecciones aprendidas, instrucción y entrenamiento, ciencia y tecnología, de tal manera que se reconfiguró el Plan Estratégico del Ejército Nacional y, de su mano, el Proyecto Educativo Institucional.

Es así como a partir del año 2016 la institución adoptó una educación constructivista y, en consecuencia, la aplicación de los diferentes modelos de enseñanza que soportan esta corriente pedagógica y que motivan el pensamiento crítico, lógico y científico. En la figura 1 se presenta un comparativo de los enfoques educativos.

Después de 2016, el enfoque pedagógico se fortaleció con estrategias motivacionales empleadas por los docentes a partir de la implementación de la educación constructivista. De este modo se promovió el aprendizaje hacia el logro de objetivos personales, que, en el marco de la formación militar, les permitiera proyectarse como profesionales integrales, conocedores de sus capacidades militares específicas y de otras propias de su formación complementaria en otras áreas del conocimiento. Así, se fue incluyendo el debate como eje a 


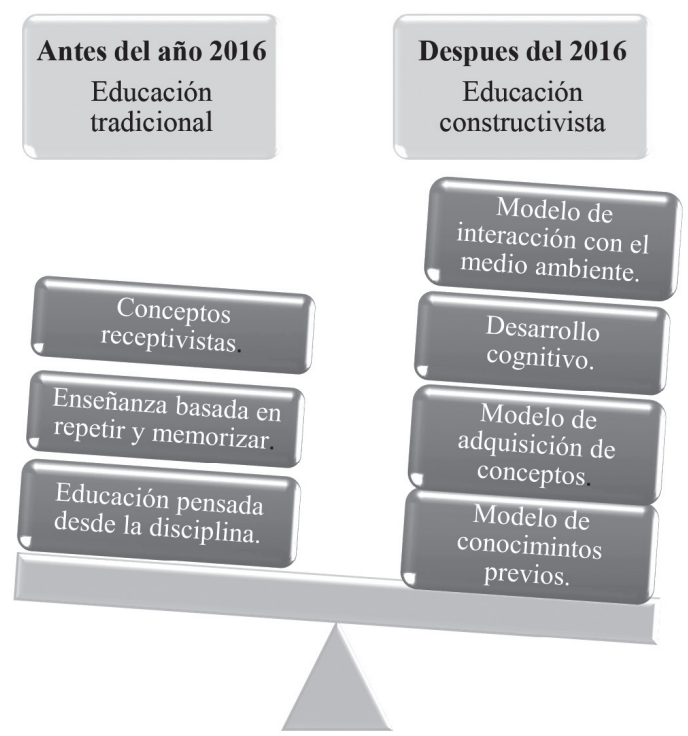

Figura 1. Comparativo de estrategias pedagógicas.

Fuente: Elaboración propia con base en Limas (2000) y Salazar-Jaramillo (s. f.).

partir del cual los cadetes y alféreces, basados en conocimientos previos, participaban activamente de las clases y contemplaban las posibilidades de llevar a la práctica lo aprendido. Se fomentó también el reconocimiento al trabajo bien hecho, se incluyó el juego como práctica para la aprehensión del conocimiento y se involucró lo aprendido en inglés como herramienta de búsqueda en investigación formativa.

\section{Segunda categoría: Competencias investigativas}

Este giro que tuvo el PEP condujo a que los cadetes y alféreces se fortalecieran en la categoría "Competencias investigativas", pues les permitió comprender el significado, la importancia y las implicaciones de la investigación en el contexto militar; observar, preguntar, registrar, interpretar, analizar y describir contextos y textos acerca de situaciones problemáticas propias de los ambientes de aprendizaje; proponer soluciones a los problemas detectados utilizando adecuadamente los conceptos y métodos de investigación; argumentar las relaciones que se establecen en el contexto militar y las alternativas 
dadas a los problemas investigados, y perfeccionar las prácticas de la escritura que contribuyan a sistematizar los datos y la información para presentarlos a través de documentos que evidencien una estructura organizada, de acuerdo con los parámetros del método científico, como el trabajo extenso de su tesis de grado o la construcción de artículos científicos.

\section{Tercera categoría: Aprendizaje significativo}

En esta categoría se evidenció una evolución satisfactoria, no solo por el número de trabajos de grado culminados, sino también por su calidad. Esto permitió que en el año 2019 se reconocieran tres trabajos de grado meritorios y uno laureado (tabla 2).

Tabla 2. Cantidad de trabajos de grado por año versus distinciones

\begin{tabular}{ccccccccc}
\hline Año & $\mathbf{2 0 1 3}$ & $\mathbf{2 0 1 4}$ & $\mathbf{2 0 1 5}$ & $\mathbf{2 0 1 6}$ & $\mathbf{2 0 1 7}$ & $\mathbf{2 0 1 8}$ & $\mathbf{2 0 1 9}$ & $\mathbf{2 0 2 0}$ \\
\hline $\begin{array}{c}\text { Número } \\
\text { de trabajos } \\
\text { presentados }\end{array}$ & 13 & 15 & 19 & 17 & 26 & 17 & 49 & 15 \\
\hline $\begin{array}{c}\text { Concepto } \\
\text { aprobado }\end{array}$ & 13 & 15 & 19 & 17 & 26 & 17 & 45 & 15 \\
\hline $\begin{array}{c}\text { Concepto } \\
\text { meritorio }\end{array}$ & 0 & 0 & 0 & 0 & 0 & 0 & 3 & 0 \\
\hline $\begin{array}{c}\text { Concepto } \\
\text { laureado }\end{array}$ & 0 & 0 & 0 & 0 & 0 & 0 & 1 & 0 \\
\hline
\end{tabular}

Fuente: Elaboración propia.

Otra forma de evidenciar el aprendizaje significativo se observó en la participación voluntaria de los estudiantes en los semilleros de investigación. Es así como desde el 2016 se observó una mayor participación de los cadetes y alféreces en eventos académicos interinstitucionales, en los cuales presentaron los resultados de investigación producto de sus trabajos de grado. De igual forma, se evidenció que las competencias investigativas adquiridas permitieron la publicación de artículos en revistas nacionales e internacionales (tabla 3). 
Tabla 3. Vinculación de estudiantes a los semilleros de investigación del grupo de investigación en Rendimiento Físico Militar (RenfimiL) versus productos significativos

\begin{tabular}{ccccccccc}
\hline Año & $\begin{array}{c}\text { N. o de es- } \\
\text { tudiantes } \\
\text { semillero } \\
\text { Citius }\end{array}$ & $\begin{array}{c}\text { Pro- } \\
\text { yectos } \\
\text { desarro- } \\
\text { llados }\end{array}$ & $\begin{array}{c}\text { Publica- } \\
\text { ciones en } \\
\text { revistas de } \\
\text { difusión }\end{array}$ & $\begin{array}{c}\text { Ponen- } \\
\text { cias } \\
\text { nacio- } \\
\text { nales }\end{array}$ & $\begin{array}{c}\text { N. o de es- } \\
\text { tudiantes } \\
\text { semillero } \\
\text { InciensA }\end{array}$ & $\begin{array}{c}\text { Proyectos } \\
\text { desarro- } \\
\text { llados }\end{array}$ & $\begin{array}{c}\text { Publica- } \\
\text { ciones en } \\
\text { revistas de } \\
\text { difusión }\end{array}$ & $\begin{array}{c}\text { Ponen- } \\
\text { cias } \\
\text { nacio- } \\
\text { nales }\end{array}$ \\
\hline 2016 & 24 & 7 & 2 & 5 & 6 & 3 & 0 & 0 \\
\hline 2017 & 24 & 8 & 3 & 8 & 6 & 3 & 0 & 3 \\
\hline 2018 & 14 & 5 & 4 & 2 & 8 & 4 & 2 & 2 \\
\hline 2019 & 19 & 4 & 6 & 4 & 8 & 3 & 0 & 3 \\
\hline 2020 & 21 & 4 & 1 & 1 & 8 & 2 & 2 & 1 \\
\hline
\end{tabular}

Fuente: Elaboración propia.

A partir de la obtención de la acreditación de alta calidad, mediante la Resolución 17148 del 27 de diciembre de 2012, por siete años, modificada por la Resolución 19478, y acreditado mediante Resolución 15488 del 30 de noviembre de 2012, por seis años, la Facultad de Educación Física Militar se propone como misión promover la investigación en los temas relacionados con la educación física y el deporte militar en sus aspectos formativo y competitivo, con el fin de generar y compartir conocimiento con la comunidad académica y científica. Así, surgen las áreas de formación en investigación, a saber: Capacidades Físicas, Biomecánica, Análisis de Movimiento, Fisiología del Ejercicio y Entrenamiento Deportivo I y II.

Desde cada área se trabajaba en competencias investigativas comunes, como son: el análisis de situaciones complejas, curiosidad intelectual, actitud creadora e innovadora, trabajo en equipo, expresión oral y escrita, y evaluación, las cuales fortalecen las competencias mencionadas en el ítem anterior. En este contexto, los estudiantes que se iniciaban en la formación pasaban por tres niveles: el primero, denominado Capacitación, hacía partícipes a los estudiantes de talleres y seminarios donde aprendían sobre la investigación aplicada a la actividad física y el deporte. En segundo lugar, en el nivel Proyección, 
creaban su idea de investigación y realizaban intercambio de experiencias con semilleros de otras instituciones.

En el tercer y último nivel, Aplicación, se formalizaba la participación en un proyecto, liderado por un docente, y el cadete o alférez cumplía con sus compromisos como asistente-coinvestigador. A partir de esta experiencia se lograron valiosos resultados: desde el año 2013 aumentaron progresivamente el número de participantes en los semilleros progresivamente y la difusión de los resultados en revistas científicas y en ponencias nacionales, aspectos que evidencian el éxito en la adquisición de las competencias investigativas.

\section{Cuarta categoría: Docentes}

Finalmente, en esta categoría se aprecia que el perfil docente fue cada vez más exigente, lo cual, sin duda, ha contribuido en el proceso de enseñanza-aprendizaje en investigación formativa. En este punto es importante mencionar que, en la actualidad, la interdisciplinariedad del cuerpo docente también ha permitido comprender el objeto de estudio del programa, desde una perspectiva más amplia, con mayores posibilidades de abordar las problemáticas de investigación y con una mirada integradora. En la tabla 4 se presenta cronológicamente la evolución del perfil docente.

Tabla 4. Evolución del nivel de formación profesional en los docentes de la Facultad de Educación Física Militar

\begin{tabular}{lcccccccc}
\hline \multicolumn{1}{c}{ Año } & $\mathbf{2 0 1 3}$ & $\mathbf{2 0 1 4}$ & $\mathbf{2 0 1 5}$ & $\mathbf{2 0 1 6}$ & $\mathbf{2 0 1 7}$ & $\mathbf{2 0 1 8}$ & $\mathbf{2 0 1 9}$ & $\mathbf{2 0 2 0}$ \\
\hline $\begin{array}{l}\text { Cantidad } \\
\text { de docentes } \\
\text { vinculados }\end{array}$ & 18 & 21 & 21 & 22 & 23 & 24 & 25 & 25 \\
\hline $\begin{array}{l}\text { Nivel } \\
\text { especialización }\end{array}$ & 16 & 14 & 10 & 7 & 6 & 5 & 5 & 5 \\
\hline $\begin{array}{l}\text { Nivel maestría } \\
\text { Nivel }\end{array}$ & 2 & 4 & 11 & 15 & 17 & 19 & 19 & 18 \\
\hline doctorado & 0 & 0 & 0 & 0 & 0 & 0 & 1 & 2 \\
\hline
\end{tabular}

Fuente: Elaboración propia. 


\section{Discusión}

En los últimos cinco años se ha evidenciado que el proceso de enseñanza-aprendizaje ha venido optimizando el aprendizaje significativo de competencias investigativas en los cadetes y alféreces, en estrategias de pensamiento, búsqueda de información, comparación, clasificación, análisis y escritura, así como competencias comunicativas. Competencias que han llevado a la práctica diaria, pues estas contribuyen a un mejor desempeño en las demás asignaturas y les han permitido comprender la importancia de la investigación en su formación como futuros oficiales. $\mathrm{Al}$ respecto, se ha afirmado que la investigación formativa prepara al estudiante para la comprensión de lectura científica, a través de la adquisición de habilidades en lectura interpretativa y comprensión de significados, entre otros, a través de procesos cognitivos y metacognitivos individuales y grupales (Viteri \& Vázquez, 2016).

Las estrategias pedagógicas iniciales generaban en los estudiantes una actitud pasiva como receptores de conocimientos y conceptos encauzados por el docente como único transmisor, de tal manera que se orientaba contenidos específicos sobre cada saber sin establecer interrelaciones con otras áreas del conocimiento (Nuván et al., 2018), pues el objetivo primordial consistía en repetir a la perfección el contenido aprendido. Por ello la EsMic planteó una serie de iniciativas para fortalecer la educación y la doctrina, marcando los lineamientos del desarrollo institucional a través del fortalecimiento de los subsistemas de educación, doctrina, lecciones aprendidas, instrucción y entrenamiento, ciencia y tecnología. De esta manera, en el año 2015 surgió el Plan Estratégico del Ejército Nacional (Minerva), mediante el cual se direccionaron y enfocaron los esfuerzos y recursos hacia la formación, capacitación y entrenamiento militar, y se resaltó la investigación militar como mecanismo para hacer del Ejército Nacional un ejemplo de la transformación que liderara en su momento el gobierno nacional en el sector Defensa (Rojas, 2017).

Es así como a partir del año 2016 la institución adoptó una educación constructivista y con ello la aplicación de los diferentes modelos de enseñanza que soportan dicha corriente. En el modelo de conocimientos previos de Ausubel, citado por Limas (2000), aprender significa que los nuevos aprendi- 
zajes conectan con los anteriores, enlazándose para crear un nuevo significado (Viera, 2003).

Desde la concepción constructivista del aprendizaje, se afirma que al ubicar al estudiante como el impulsor de su propio aprendizaje, guiado por la acción mediadora del docente, es posible promover nuevas maneras de investigar y enseñar (Carranza, 2017). Por ello, en la Facultad de Educación Física Militar se promueve la actualización docente permanente, que va más allá de lo disciplinar e involucra la adquisición de nuevas estrategias motivacionales que dinamicen la enseñanza, promuevan la reflexión y conduzcan al desarrollo de habilidades investigativas, que no solo contribuyan en su producción científica, sino que además los doten de habilidades para enseñar a investigar a sus estudiantes, pues como afirma Viteri y Vásquez (2016), cuando el profesor actualiza sus conocimientos y prácticas, insta al estudiante a involucrarse en estos procesos, con lo cual genera hábitos y habilidades de investigación formativa (Viteri \& Vázquez, 2016).

En el aprendizaje significativo es necesario que los estudiantes, al sentirse motivados, se apropien del proceso, de tal manera que ellos mismos trabajen en su autoformación, pues el deseo por aprender los hará enfocarse desde sus propias realidades individuales. Por lo tanto, es vital que en el proceso se tomen en cuenta sus aspiraciones y deseos, aprovechándolos como estrategias motivacionales hacia la búsqueda de nuevos aprendizajes, de tal manera que el proceso, al cobrar sentido y significado, les confiera satisfacción (Yáñez, 2016).

Desde la mirada de Valenzuela et al. (2018), la motivación debe articularse en todo proceso educativo, pues de ella depende el deseo por aprender. Así las cosas, estos autores clasifican las estrategias motivacionales en tres: las estrategias motivacionales hacia el aprendizaje, las estrategias para promover la realización de tareas y las estrategias para hacer de la motivación una entretención. Dentro de dichas estrategias se resalta el planteamiento de interrogantes, como garantes en la generación de debates que permiten la activación de los conocimientos previos de los estudiantes y el establecimiento de relaciones entre lo que se enseña y el mundo real. Asimismo, la orientación hacia el proceso, más que hacia el resultado, la visualización del aprendizaje en un ambiente de disfrute y el uso de materiales y recursos novedosos, entre otros, 
favorecerán el aprendizaje significativo (Valenzuela et al., 2018), todo lo cual se evidencia en la evolución que ha tenido el proceso de enseñanza-aprendizaje del programa de Educación Física Militar.

\section{Conclusiones}

El Programa de Educación Física Militar, desde la obtención de registro calificado en 2008 y posterior acreditación de alta calidad en 2012 hasta la fecha, ha asumido un reto importante en la investigación formativa que se evidencia en los ajustes que ha hecho a las estrategias didácticas, las cuales han contribuido a generar un aprendizaje significativo en investigación. Es necesario hacer este tipo de estudios para identificar las fortalezas, oportunidades, debilidades y amenazas que el proceso conlleva, a fin de obtener mejores resultados.

\section{Referencias}

Bonilla-Castro, E., \& Rodríguez Sehk, P. (2005). Más allá del dilema de los métodos. Norma.

Borja Santillán, M. A., Vásquez Portugal, R. M., \& Zeballos Chang, J. M. (2017). La filosofía analítica: Su enfoque hacia el proceso de enseñanza-aprendizaje. Sophia, (22), 149-169. http://dx.doi.org/10.17163/soph.n22.2017.06

Cabrera Cabrera, L. J., \& Bernal, V. R. (2018). Historia y educación militar para el futuro: Investigación formativa. Sello Editorial ESMIC. https://doi. org/10.21830/9789585989665

Carranza Alcántar, M. (2017). Enseñanza y aprendizaje significativo en una modalidad mixta: Percepciones de docentes y estudiantes. RIDE. Rev. Iberoam. Investig. Desarro. Educ, 8(15), 898-922. https://doi.org/10.23913/ride.v8i15.326

Consejo de Facultad de Educación Física Militar. (2019). Proyecto educativo del programa "Profesional en educación física militar".

Escuela Militar de Cadetes "General José María Córdova” [ESMIC]. (2016). Acuerdo 008 de 2016. "Por medio del cual se aprueba y adopta el proyecto educativo institucional".

Hernández Sampieri, R., Fernández Collado, C., \& Baptista Lucio, M. (2010). Metodología de la investigación. McGrawHill.

Limas Santiváñez, V. (2000). La didáctica, el constructivismo y su aplicación en el aula. Rev. Cultura, 18(8), 137-148. http://www.revistacultura.com.pe/revistas/RCU_18_1_la-didactica-el-constructivismo-y-su-aplicacion-en-el-aula.pdf 
Lizarazo Caparo, F., \& Paredes Pérez, N. (2015). Proceso enseñanza aprendizaje: Desde la sociedad para servir a la sociedad. Horiz. Med, 15(3), 4-5.

Morales-Ocańa, A., \& Higueras-Rodríguez, M. L. (2017). Procesos de enseñanza-aprendizaje: Estudios, avances y experiencias. Profesorado. Revista de Currículum y Formación de Profesorado, 21(2), 1-6.

Nuván Hurtado, I. L., Rivera Porras, D. A., Carrillo Sierra, S. M., Fiorgiony Oreste, S., Bonilla Cruz, N. J., \& Rozo Sánchez, A. C. (2018). Diferencias en la calidad psicométrica de test construidos mediante la estrategia pedagógica audiovisual y las estrategias pedagógicas tradicionales. Espacios, 39(25), 7-28.

Parra F., K. N. (2014). El docente y el uso de la mediación en los procesos de enseñanza y aprendizaje. Revista de Investigación, 38(83), 155-180.

Rojas Guevara, P. J. (2017). Doctrina Damasco: Eje articulador de la segunda gran reforma del Ejército Nacional de Colombia. Revista Cientifica General José María Córdova, 15(19), 95- 119. http://dx.doi.org/10.21830/19006586.78

Salazar-Jaramillo, R. (s. f.). Pedagogía tradicional versus pedagogía constructivista: Repetir un saber ¡No!, construirlo ¡Sí! https://www.flacsoandes.edu.ec/sites/default/files/agora/ files/1394726224.salazarr_pt_vs_pc.pdf

Valenzuela, J., Muñoz, C., \& Montoya Levinao, A. (2018). Estrategias motivacionales efectivas en profesores en formación. Educ. Pesqui, (44), 1-20. http://dx.doi.org/10.1590/s1678$4634201844179652 \mathrm{E}$

Viera Torres, T. (2003). El aprendizaje verbal significativo de Ausubel: Algunas consideraciones desde el enfoque histórico cultural. Universidades, (26), 37-43. https://www.redalyc.org/ pdf/373/37302605.pdf

Viteri Briones, T. A., \& Vázquez Cedeño, S. (2016). Formación de habilidades de investigación formativa en los estudiantes de la carrera de Ingeniería Comercial de la Facultad de Ciencias Administrativas de la Universidad de Guayaquil. Revista Universidad y Sociedad, 8(1), 36-44.

Yánez M., P. (2016). El proceso de aprendizaje: Fases y elementos fundamentales. Revista San Gregorio, 1(11), 70-81. 